\title{
Pulmonary cryptococcosis in an immunocompetent patient-A case report
}

\author{
Fernando Morbeck*, Rafael Franco, Sergio Furlan, Marcos Duarte \\ Department of Radiology, Heliopolis Hospital, São Paulo, Brazil; ${ }^{*}$ Corresponding Author: dr.fernandomorbeck@gmail.com
}

Received 23 October 2013; revised 20 November 2013; accepted 11 December 2013

Copyright (c) 2013 Fernando Morbeck et al. This is an open access article distributed under the Creative Commons Attribution License, which permits unrestricted use, distribution, and reproduction in any medium, provided the original work is properly cited. In accordance of the Creative Commons Attribution License all Copyrights @ 2013 are reserved for SCIRP and the owner of the intellectual property Fernando Morbeck et al. All Copyright (C) 2013 are guarded by law and by SCIRP as a guardian.

\begin{abstract}
Cryptococcosis is an infection caused by fungi that belong to the genus Cryptococcus. There are several species of Cryptococcus, but two species-Cryptococcus neoformans and Cryptococcus gattii-cause nearly all cryptococcal infections in humans. Cryptococcosis is one of the most common fungal infections in Brazil and becomes even more important after the onset of the AIDS epidemic. The lung is the main gateway, and also is the most common site, with diverse clinical manifestations ranging from an asymptomatic to severe pneumonia. When symptommatic, most patients present with fever and cough with expectoration or hemoptysis. The most common radiological findings in immunocompetent patients are located images of masses and nodules, while immunosuppression shows interstitial infiltrates and diffuses interstitial opacity. This is a case report of a patient in the third decade of life with breathing-dependent pain at the base of the left hemithorax, who has radiological examination of a mass in the left lung base in contact with the pleura mimicking malignant lung. Lobectomy was performed, as well as the pathological diagnosis of cryptococcosis.
\end{abstract}

Keywords: Cryptococcosis; Pulmonary; Immunocompetent; Fungal; Lung Disease

\section{INTRODUCTION}

Cryptococcosis (Busse-Buschke disease) is a fungal disease caused by Cryptococcus neoformans and Cryptococcus gattii. The first is an opportunistic disease, cosmopolitan and conditions associated with immunosuppression cell. It occurs in various organic substrates, and is usually rich in nitrogen source such as from bird feces. Microfoci's growth of this yeast is formed mainly in urban centers and related to pigeons. C. gattii is related to infection of immunocompetent patients, being endemic in tropical and subtropical areas. Its natural habitat primarily is related to Eucalyptus camaldulensis plant remains from Australia, but has been isolated from different types of wood elsewhere in the world [1].

The mortality of the disease varies with the degree of development of the country. In developed regions, the rate is approximately $10 \%$ and around $43 \%$ in developing countries. In the Northeast and North regions of Brazil, C. gatti infection of the central nervous system has lethality of approximately $35 \%$ to $40 \%$. In the South Region, Southeast and Midwest, diseases secondary to $C$. neoformans are prevalent because there is a higher rate of AIDS [1].

Inhalation of dried yeasts causes natural infection, causing an initial framework lung, which may progress or regress depending on the host response. The clinical picture may reach beyond the lung lesions, skin, bones, adrenal, kidney and central nervous system, among others [2].

Pulmonary infection is the most frequent, and the clinical and radiological spectrum is quite broad. The immune status of the patient plays an important role in this range of signs and symptoms, and immunocompetent patients tend to present with more self-limiting localized lesions, since they are immunosuppressed with dissemination and respiratory failure. In one-third of cases, the disease is asymptomatic. The symptoms are very nonspecific, predominantly fever and cough [3]. The involvement of local lesions causes specific symptoms: breathing-dependent pain (mass contact with the pleura) and the upper lobes (mimics the Pancoast syndrome). Furthermore, constitutional symptoms may be present, with the union of clinical factors-radiologically indistinguishable from malignant lung injury. 
Unilateral or bilateral nodules are the most common, and are rarely calcified or cavitary on radiological examination. They generally stay on subpleural topography with diameter ranging from 0.5 to $4.0 \mathrm{~cm}$ and are characteristic of immunocompetent patients. Already, immunosuppressed patients have interstitial infiltrates and must always make the differential diagnosis with pneumocystosis. There is also the miliary and hilar and mediastinal lymphadenopathy similar to tuberculosis. Pleural effusion is present in less than $10 \%$ of the cases [1].

The diagnosis of an immunocompetent patient with pulmonary involvement is usually done by biopsy or surgical removal of the nodule with its histopathology. If confirmed, there is a need to investigate infection of the central nervous system via lumbar puncture.

The treatment of symptomatic patients with disseminated disease, positive cryptococcal antigen or immunosuppression should be performed with anti-fungal. However, those with localized, oligosymptomatic or asymptomatic pulmonary forms or patients with resected pulmonary nodules [1] can be accompanied by rigorous clinical and laboratory monitoring. The surgery for resection of the lesion is performed in specific cases such as pseudotumor injuries and not responsive to medical treatment, and extensive exudative pleural effusion.

\section{CASE REPORT}

A 36-year-old african american woman with no significant past medical history was attended at Heliopolis Hospital Emergency Room (Sao Paulo - Brazil) due to a breathing-dependent pain in the left hemithorax from one week. Five days prior, the patient sought another service being prescribed antibiotic therapy, but there was no improvement. The physical examination revealed decreased breath sounds in the lower third of the left hemithorax and vital signs unchanged.

During the diagnostic investigation, the patient had hemoglobin of $10.5 \mathrm{mg} / \mathrm{dL}$, white blood cell $8720 \mathrm{mg} / \mathrm{dL}$, C-reactive protein $6.1 \mathrm{mg} / \mathrm{dL}$, and the HIV virus search was negative.

Chest radiograph (Figures 1 and 2) presents extensive opacity in the lower third of the left hemithorax in the posterior region. The patient was admitted for investigation.

Computed tomography (Figure 3) showed low attenuation mass, heterogeneous, suggesting areas of necrosis in the lower lobe in lateral and posterior basal segment associated with ipsilateral pleural effusion.

A thoracentesis was performed, looking bloody, with blood glucose of $116 \mathrm{mg} / \mathrm{dL}$, LDH $838 \mathrm{U} / \mathrm{L}$, albumin 3.8 $\mathrm{g} / \mathrm{dL}$, amylase $36 \mathrm{U} / \mathrm{L}$ and negative culture in the first sample. The cytology was negative. A second thoracentesis was attempted, but not enough liquid was evidenced

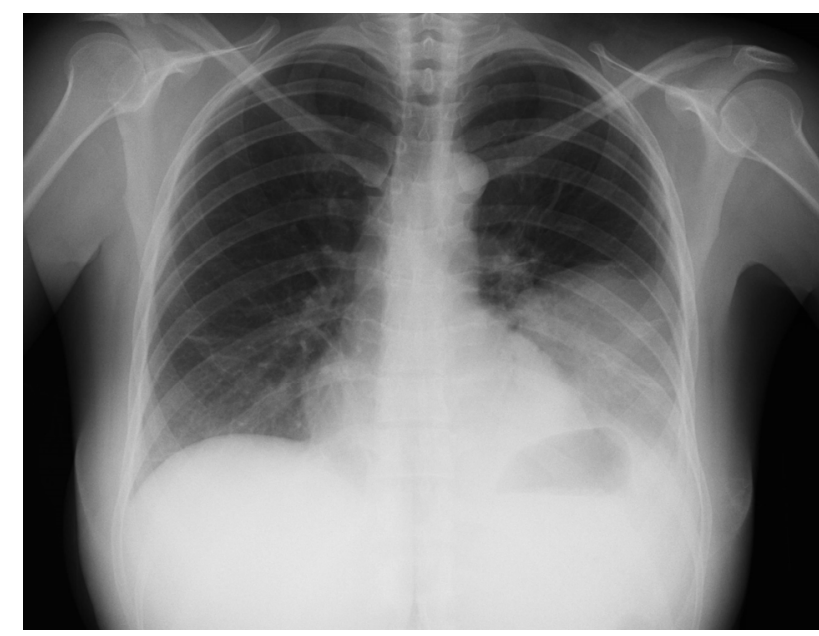

Figure 1. Chest X-ray.

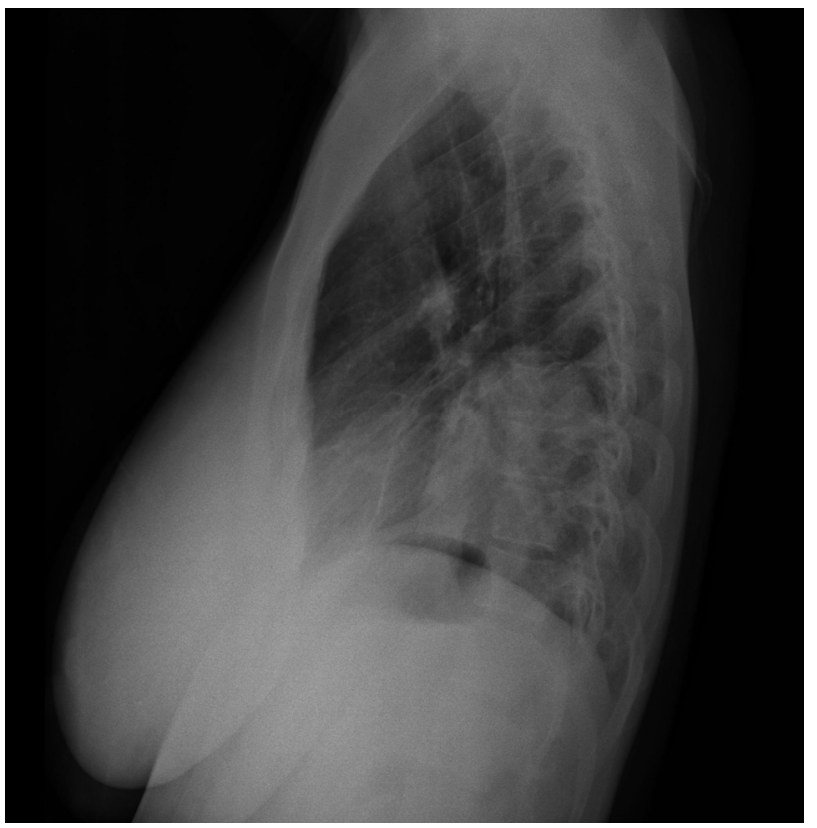

Figure 2. Chest X-ray.

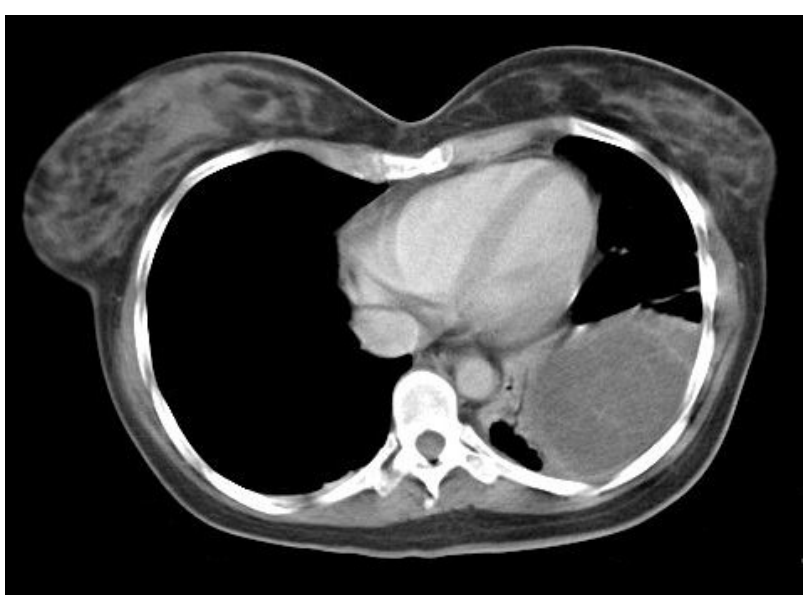

Figure 3. Computerized Tomography (CT). 
by ultrasound.

Diagnostic option was surgical resection of the tumor which, under direct visualization, had hardened aspect and was adhered to the chest wall and diaphragm. A lobectomy was performed. The pathological analysis was positive for cryptococcal infection (Figure 4).

\section{DISCUSSION}

Cryptococcal infection presents an increased prevalence in patients with immunosuppression of $\mathrm{T}$ cells. The male is the most affected. In patients with HIV, the radiological pattern is a predominant interstitial infiltration, alveolar infiltrates, mixed infiltrate and pleural effusion. A less common finding is excavation. Immunocompetent individuals, such as the patient in question, present with nodular lesions or masses [1,4]. Other cases of immunocompetent patients reported in the literature showed the same image aspect, however, clinical history was variable $[4,6,7]$.

Regarding the aspect of injury, the lung mass with irregular delimited contours, more prevalent in radiology, is often associated with close contact with the pleural surface. Pulmonary nodules also exhibit irregular defined contours [4,5]. Involvement is most often unilateral. A study of 14 patients with a diagnosis of pulmonary cryptococcosis confirmed by cytological and histological investigation showed that $64.2 \%$ of the lesions had the mass aspect and $50 \%$ had contact with the pleura [4].

The radiological finding of an extensive lesion in the lung with irregular contours suggests malignancy, but the

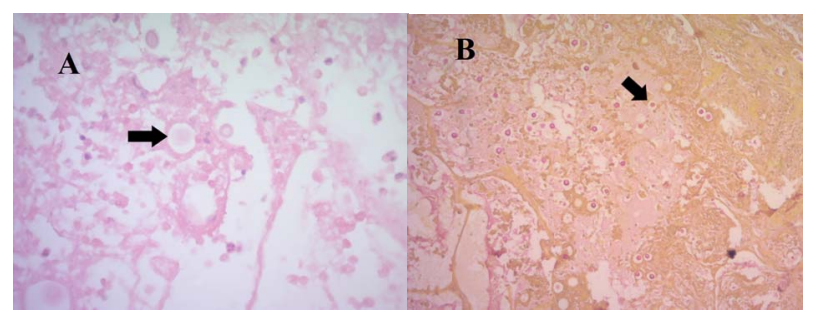

Figure 4. (A) Histological examination with hematoxylin and eosin showed the presence of spore (arrow). (B) Mucicarmine staining (arrow) confirmed the diagnosis of Cryptococcus. differential diagnosis with infection, infarction, and abscess should be performed. The use of PET/CT has an indeterminate finding, because both malignancies such as infection and inflammation are often hypermetabolic. These findings cause many false positives in endemic areas [5]. Surgical treatment should not be the first option in cases of cryptococcal infection. However, when it does not respond adequately to drug therapy, a resection of the lesion (usually a lobectomy) should be performed.

This report reinforces the importance of pseudotumoral lesions for the differential diagnosis of lung masses by radiologists, pulmonologists and oncologists.

\section{REFERENCES}

[1] Kon, A.S., Grumach, A.S. and Colombo, A.L., et al. (2008) Guidelines in cryptococcosis. Revista da Sociedade Brasileira de Medicina Tropical, 41, 524-544.

[2] Patz Jr., E.F. and Goodman, P.C. (1992) Pulmonary cryptococcosis. Journal of Thoracic Imaging, 7, 51-55. http://dx.doi.org/10.1097/00005382-199209000-00008

[3] Severo, C.B., Gazzoni, A.F. and Severo, L.C. (2009) Pulmonary cryptococcosis. Jornal Brasileiro de Pneumologia, 35, 1136-1144. http://dx.doi.org/10.1590/S1806-37132009001100012

[4] Silva, A.C.G., Marchiori, E., Souza Jr., A. and Irion, K.L. (2003) Criptococose pulmonar: Aspectos na tomografia computadorizada. Radiologia Brasileira, 36, 277-282. http://dx.doi.org/10.1590/S0100-39842003000500005

[5] Guimarães, M.D., Marchiori, E., Meirelles, G.S.P., et al. (2013) Fungal infection mimicking pulmonary malignnancy: Clinical and radiological characteristics. Lung, in press. http://dx.doi.org/10.1007/s00408-013-9506-0

[6] Fong, I.A., Glória, C., Mesquita, I. and Diogo, N. (1999) Pulmonary cryptococcosis in an immunocompetent patient. A case report and a review. Revista Portuguesa de Pneumologia, 4, 419-426.

[7] Barbosa, A.T.F., Colares, F.A., Gusmão, E.S., Barros, A.A., Cordeiro, C.G. and Andrade, M.C.T. (2006) Isolated pulmonary cryptococcosis in an immunocompetent patient. Jornal Brasileiro de Pneumologia, 32, 470-480. http://dx.doi.org/10.1590/S1806-37132006000500016 